\title{
Biochemical routes for uptake and conversion of xylose by microorganisms
}

Zhe Zhao ${ }^{1,2}$, Mo Xian ${ }^{1}$, Min Liu ${ }^{1 *}$ and Guang Zhao ${ }^{\text {** }}$

\begin{abstract}
Xylose is a major component of lignocellulose and the second most abundant sugar present in nature. Efficient utilization of xylose is required for the development of economically viable processes to produce biofuels and chemicals from biomass. However, there are still some bottlenecks in the bioconversion of xylose, including the fact that some microorganisms cannot assimilate xylose naturally and that the uptake and metabolism of xylose are inhibited by glucose, which is usually present with xylose in lignocellulose hydrolysate. To overcome these issues, numerous efforts have been made to discover, characterize, and engineer the transporters and enzymes involved in xylose utilization to relieve glucose inhibition and to develop recombinant microorganisms to produce fuels and chemicals from xylose. Here we describe a recent advancement focusing on xylose-utilizing pathways, biosynthesis of chemicals from xylose, and engineering strategies used to improve the conversion efficiency of xylose.
\end{abstract}

Keywords: Xylose, Lignocellulose, Xylose transporter, Xylose catabolic pathways, Escherichia coli, Saccharomyces cerevisiae, Carbon catabolite repression, Chemicals produced from xylose

\section{Background}

With the increasing concerns regarding the energy crisis and global climate change, it has become of utmost importance to develop clean and sustainable sources for the production of fuels and chemicals. Lignocellulosic biomass is an environment-friendly alternative to fossil energy for fuels and chemicals production and has attracted a great deal of attention, because it is considered the most abundant and non-food-oriented resource. This biomass is a renewable resource by solar energy and carbon dioxide fixation $[1,2]$. Lignocellulose has an intricate tridimensional network structure and is composed basically of cellulose (30-50\%), hemicellulose (25-30\%), and lignin (15-20\%). Cellulose is a homopolymer of glucose units interconnected by $\beta 1 \rightarrow 4$ glycosidic bonds, and hemicellulose is a complex branched polysaccharide

\footnotetext{
*Correspondence: liumin@qibebt.ac.cn; zhaoguang@qibebt.ac.cn

${ }^{1}$ CAS Key Laboratory of Biobased Materials, Qingdao Institute

of Bioenergy and Bioprocess Technology, Chinese Academy of Sciences, Qingdao 266101, China

Full list of author information is available at the end of the article
}

composed of xylose, arabinose and galactose, among others [3]. Conversion of lignocellulose to biofuels and chemicals requires three main steps: destroying the lignocellulose structure by pretreatment, hydrolyzing hemicellulose/cellulose to fermentable monomeric sugars by enzymatic saccharification, and finally converting monomeric sugars to chemicals or biofuels by microbial fermentation. A major issue in this process is that the majority of fermenting microorganisms cannot metabolize the degradation products of lignocellulose effectively other than glucose [3].

Xylose, derived from hemicellulose, is the second most common sugar in nature and accounts for $18-30 \%$ of lignocellulose hydrolysate sugars [4]. Therefore, xylose is considered to be a promising renewable resource for producing biofuels and chemicals. However, to date, there are still some bottlenecks in the process of xylose utilization, resulting in few varieties of products and low conversion efficiency from xylose. First, only a small fraction of microorganisms have the native xylose metabolic pathway [5]. This may greatly limit the selection of engineering strains and the application of xylose. Second, xylose

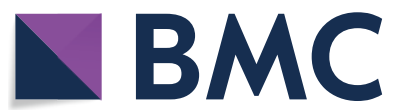

(c) The Author(s) 2020. This article is licensed under a Creative Commons Attribution 4.0 International License, which permits use, sharing, adaptation, distribution and reproduction in any medium or format, as long as you give appropriate credit to the original author(s) and the source, provide a link to the Creative Commons licence, and indicate if changes were made. The images or other third party material in this article are included in the article's Creative Commons licence, unless indicated otherwise in a credit line to the material. If material is not included in the article's Creative Commons licence and your intended use is not permitted by statutory regulation or exceeds the permitted use, you will need to obtain permission directly from the copyright holder. To view a copy of this licence, visit http://creativeco mmons.org/licenses/by/4.0/. The Creative Commons Public Domain Dedication waiver (http://creativecommons.org/publicdomain/ zero/1.0/) applies to the data made available in this article, unless otherwise stated in a credit line to the data. 
metabolism is severely repressed by glucose in many microorganisms, a process known as carbon catabolite repression (CCR). CCR is a well-known global regulatory phenomenon that allows cells to utilize the most energyefficient carbon source in a mixture, for example, glucose [6]. This leads to diauxic growth phenomena that affects the metabolism of the secondary sugars derived from lignocellulosic biomass (e.g., xylose) and further limits the conversion efficiency during the fermentation process. Third, transmembrane transport is the first key step for xylose utilization. It has been revealed in some previous studies that the transport rate of xylose limits the downstream pathway flux and that enhancing the transport rate is necessary for improving the cell growth rate and the xylose conversion efficiency [7-9].

Therefore, it is of crucial importance to improve the production of fuels and chemicals from xylose by increasing the xylose uptake efficiency and establishing an effective metabolic pathway. Here, we discuss the aspects regarding the structure and regulation of native xylose transport and catabolism systems in microorganisms as well as the establishment of artificial pathways by introducing heterologous genes. Furthermore, we present strategies to improve the efficiency of xylose uptake and metabolism, such as enzyme engineering and redox balancing. Additionally, some fuels and chemicals produced from xylose by engineered microorganisms and their corresponding pathways are also introduced.

\section{Xylose uptake}

Substrate transport is the first key step in the whole metabolism system during the conversion from lignocellulose to chemicals or fuels. Native sugar transporters, mainly including the ATP-binding cassette (ABC) transporter, the major facilitator superfamily (MFS), and the phosphoenolpyruvate (PEP):carbohydrate phosphotransferase system (PTS), present distinctive substrate specificity and transport mechanism [10]. For example, small soluble molecules are usually transported by the singlepolypeptide secondary carriers of MFS with the action of the ion gradient. The MFS is divided into 17 families, and the sugar transport includes families 1,5 , and $7[11,12]$. Different from the above type, one molecule of ATP per sugar is needed by the $A B C$ transporter, and the phosphate group derived from PEP is transferred to substrate sugar by PTS.

\section{Native xylose transporters}

Escherichia coli is one of the most important platform organisms because of its fast growth, clear genetic background, easy genetic manipulation, and low cultivation cost. Moreover, E. coli has the native transporters and metabolism pathways of xylose, which confers the ability to use mixed sugars derived from lignocellulosic biomass [13]. Even so, the existence of CCR makes E. coli cells preferentially metabolize glucose over xylose [6].

The native xylose transporters of E. coli mainly include the MFS protein XylE and the ABC transporter XylFGH. However, the arabinose symporter (AraE) can also play the role of xylose transport in some special situations [14]. The XylE protein is a D-xylose/proton symporter with a relatively low affinity, and the $K_{\mathrm{m}}$ value for xylose is between 63 and $169 \mu \mathrm{M}[13,15]$. Xylose transport by XylE is driven by a proton motive force rather than a direct energy drive or a phosphotransferase, because the addition of xylose to energy-consuming $E$. coli cells can elicit an obvious change of alkaline $\mathrm{pH}$. However, the alkaline $\mathrm{pH}$ change fails to appear by uncoupling agents. The uncoupling agents also inhibit the accumulation of $\left[{ }^{14} \mathrm{C}\right]$ xylose in energy-replete cells, whereas arsenates and fluoride do not. The crystal structures of XylE with xylose or glucose (PDB no. 4GBZ) have been obtained, which may provide some valuable insights into the rational design of XylE mutants with improved activity and specificity [16].

The XylFGH protein is a high-affinity $\mathrm{D}$-xylose $\mathrm{ABC}$ transporter (the $K_{\mathrm{m}}$ value for xylose is between 0.2 and $4.0 \mu \mathrm{M})$ compared with the XylE symporter [15]. Sequence homology analysis suggested that XylF is a periplasmic xylose-binding protein, XylG is an ATP-binding protein, and $\mathrm{XylH}$ is a membrane component of the $\mathrm{ABC}$ transporter $[17,18]$. Among the above systems, XylFGH is the dominant xylose transporter in $E$. coli because the $x y l E$ knockout mutant showed a much higher growth rate than the $x y l G$ mutant when xylose was used as the sole carbon source [14].

Clostridia has been widely used for producing acetone, butanol and ethanol (ABE) because of the ability to metabolize a wide range of carbohydrates, such as hexose sugars (glucose, mannose, fructose and galactose), pentose sugars (arabinose and xylose) and disaccharides (lactose and sucrose) [19]. Clostridium beijerinckii and Clostridium acetobutylicum, the two major species of solventogenic clostridia, have the native xylose transporters and metabolism pathways. However, the xylose utilization efficiency of Clostridia is also poor. The xylose transporters of Clostridia are very similar to those of $E$. coli. The native xylose transporters of $C$. beijerinckii include the sugar-proton symporter (encoded by $x y l T$ ) and D-xylose ABC transporter (encoded by $x y l F G H$ ). However, the genes of $\mathrm{ABC}$ transporter are not detected in the chromosome of C. acetobutylicum, and only several genes (CAC1339, CAC1345, CAC1530, CAC3422 and CAC3451) encoding sugar-proton symporters are identified $[20,21]$. These above symporter genes are distributed 
in the different parts of the chromosome, and located relatively far apart from each other.

In Archaea, the sugar transporters mainly belong to the class of $A B C$ transporters. Surprisingly, the ABC transporter for D-xylose in Archaea exhibits more similarity to that of bacterium than to any characterized archaeal one. It is revealed that this pentose transporter is the first identified $\mathrm{ABC}$ transporter of the carbohydrate uptake transporter-2 (CUT2) family in the domain of Archaea. In addition, the growth defect on D-xylose in the single-gene deletion mutants of $\mathrm{ABC}$ transporter further illustrated the importance of this transport system [22]. Sulfolobus solfataricus and the closely related Sulfolobus acidocaldarius are the best-studied organisms in the phylum of crenarchaeota for sugar transport, and a number of sugar $A B C$ transporters have been identified and examined $[23,24]$. Besides those $A B C$ transporters, only the MFS transporter for glucose and PTS system for D-fructose were characterized in Haloferax volcanii and Haloarchaea, respectively [22].

\section{Transcriptional regulation of xylose transporter genes}

The transcription of the $x y l F G H$ gene, as well as the $x y l A B$ gene encoding xylose catabolic enzymes of $E$. coli, is coregulated by a cyclic AMP (cAMP) receptor protein (CRP) and XylR regulator [25]. CRP is a global regulator and exhibits pleiotropic phenotypes by forming a complex with cAMP. The CRP-cAMP complex contributes to $\mathrm{CCR}$ and positively regulates the catabolic operons for secondary sugars, such as xylose, galactose, and arabinose, only under glucose-depleted conditions [26, 27]. In order to facilitate xylose uptake and utilization in the presence of glucose, a cAMP-independent mutant CRP* has been discovered and successfully applied in xylitol production from a glucose and xylose mixture $[28,29]$. However, the CRP* mutant cannot function in all E. coli strains as it did not alleviate glucose repression in $E$. coli B strains FL06 and ATCC 11303 as in E. coli K-12 [30]. Moreover, the expression of $x y l F G H$ and $x y l A B$ genes is also positively regulated by XylR in response to the $\mathrm{D}$-xylose substrate through direct binding on the corresponding promoters [25]. Unlike XylFGH, the regulatory mechanism of XylE is poorly understood. It is proposed that XylE may also be under the regulation of CRP and XylR [31].

The xylose uptake and metabolism in Clostridia are regulated by a specific repressor XylR and a pleiotropic regulator CcpA [21]. In contrast to the regulatory role in E. coli, XylR negatively regulates expression of most genes related with xylose metabolism in C. beijerinckii, and deletion of $x y l R$ results in an obviously improvement for xylose utilization efficiency [32]. However, the $x y l R$ gene in C. acetobutylicum is less certain as compared to that of $C$. beijerinckii, and at least five candidate genes of $x y l R$ have been raised so far [21]. The expression of xylose uptake and metabolism pathway in C. acetobutylicum is directly repressed by CcpA [33]. In addition, the transcription of $x y l R$ gene is also repressed by CcpA. CcpA also plays an important role in regulating the CCR in gram-positive bacteria [33]. It is demonstrated that C. acetobutylicum can co-ferment glucose and xylose in a $c с p A$ knockout mutant. So, CcpA may be a potential engineering target for improving the ability of mixedsugar fermentation of Clostridia.

Although there are several native xylose transporters in different microorganisms, the affinity for xylose is low and actually affects the xylose uptake. Therefore, it is still a long-term and arduous task to improve the xylose transport efficiency by discovering some new native xylose transporters or engineering the known ones.

\section{Introduction of heterologous xylose transporters in Saccharomyces cerevisiae}

Saccharomyces cerevisiae is also one of most widely used industrial microorganisms. However, wild-type S. cerevisiae shows an extremely low cell growth rate with xylose as the sole carbon source due to the lack of efficient and specific xylose transporters [34]. The native hexose transporters in S. cerevisiae, which are encoded by $h x t 1-$ $h x t 7$ genes, could be considered as candidates for xylose uptake, but these candidates showed much higher affinities to glucose than to xylose [34, 35]. For example, the native high-affinity hexose transporter Hxt7 presents a $K_{\mathrm{m}}$ value for xylose of $130 \mathrm{mM}$ [35]. Fortunately, some efficient xylose transporters were found in other microorganisms. This may be a promising strategy for introducing efficient xylose transporters to the common hosts that cannot transport xylose efficiently.

Filamentous fungi are usually used for screening nonglucose transporters because they can use a multiple of monosaccharides and oligosaccharides and also have the ability to encode a variety of sugar transporters closely related to hexoses and pentoses [36]. For example, Aspergillus nidulans is predicted to encode 357 proteins belonging to the MFS, although the number of proteins actually applied to sugar transport is unknown [37]. $\mathrm{HxtB}$, previously considered as a glucose transporter in $A$. nidulans, proved to be a major xylose transporter because the deletion of $h x t B$ resulted in a remarkable decline in the xylose uptake efficiency [38], and the monosaccharide transporter XtrD turned out to have a high affinity for xylose [36]. Similarly, Trichoderma reesei is predicted to encode 164 proteins belonging to the MFS, which are also candidates for effective xylose transport [39]. Besides, the ortholog of previously identified xylose transporters can be used for further screening. For 
example, MstA from Aspergillus niger is the ortholog of HxtB, and it showed a high affinity for xylose when introduced into S. cerevisiae [40].

Until now, a plenty of pentose transporters from filamentous fungi have been successfully expressed in $S$. cerevisiae to improve the xylose uptake and cell growth rate, such as Xut1p, Xut3p, Xut4p, Xut5p, Xut6p, Xut7p, Sut1p, Sut4p, Xyp29p, AraTp, and Rgt2p from Pichia stipitis; Gxs1p and Gxf1p from Candida intermedia; XylHPp, 2D01474p, and 2C02530p from Debaryomyces hansenii; Stp2p, At5g17010p, and At5g59250p from Arabidopsis thaliana; Lat1p and Lat2p from Ambrosiozyma monospora; and An25p from Neurospora crassa $[34,41]$. Although so many transporters have been used for the uptake of xylose in S. cerevisiae, the results are still unsatisfactory. In the previous analysis, glucose inhibition was still a bottleneck that cannot be ignored in the process of xylose uptake and utilization [42].

\section{Xylose metabolic pathways}

An adequate understanding of natural xylose pathways can provide theoretical guidance for constructing efficient xylose metabolism strains. Currently, many microorganisms can naturally metabolize xylose, including Escherichia coli, yeast, filamentous fungi, Caldicellulosiruptor, Clostridia, Proteobacteria, and the domain
Archaea [43]. This review listed some typical metabolic pathways of D-xylose.

\section{Natural metabolism pathways of xylose}

In order to make full use of xylose in living environments, microorganisms have evolved distinct xylose utilization pathways over a long period of time (Fig. 1). Xylose is initially converted into xylulose by different enzymes in various microorganisms. In bacteria, isomerization of xylose to xylulose is usually catalyzed by xylose isomerase (XI) directly [44], whereas yeast and mycelial fungi need a two-step pathway employing $\mathrm{D}$-xylose reductase (XR) and xylitol dehydrogenase (XDH) [45]. In this $\mathrm{XR}-\mathrm{XDH}$ pathway, the first two reactions are usually accompanied with NADPH consumption and NADH production. In both bacteria and fungi, xylulose is further phosphorylated to xylulose-5-phosphate and then metabolized by the pentose phosphate pathway [46]. Furthermore, xylulose-5-phosphate can also be cleaved into acetylphosphate and glyceraldehyde-3-phosphate by phosphoketolase in Clostridia, such as C. beijerinckii and C. acetobutylicum [21, 47] and Lactococcus lactis [48]. In addition to these typical xylose pathways, a new xylose metabolism pathway was discovered in Caulobacter crescentus, an oligotrophic freshwater bacterium that employs $\alpha$-ketoglutarate as a key intermediate [49].

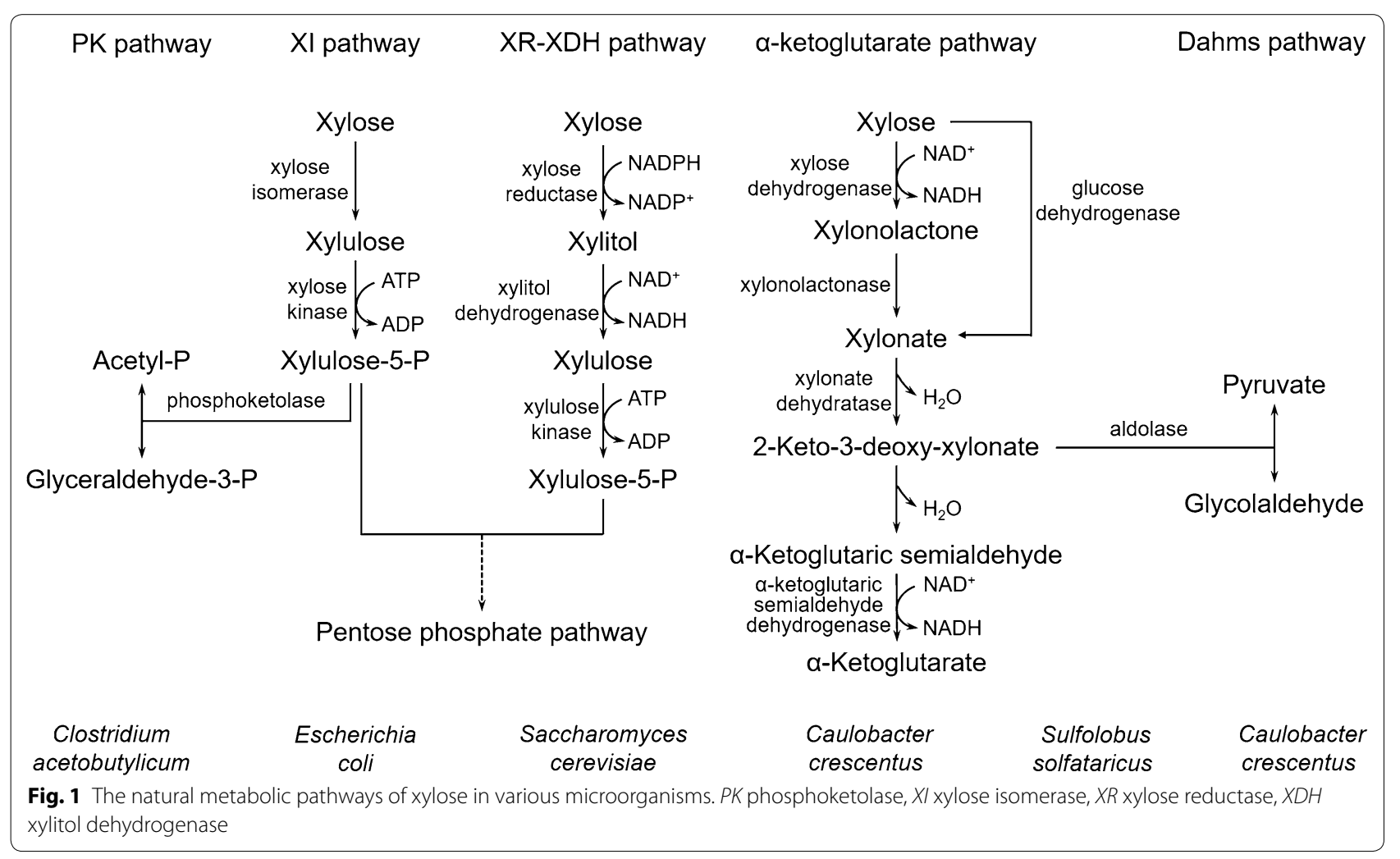


Xylose is converted into xylonolactone and xylonate by $\mathrm{NAD}^{+}$-dependent xylose dehydrogenase (Xdh) and xylonolactonase $(\mathrm{XylC})$, respectively. Then, xylonate is dehydrated into 2-keto-3-deoxy-xylonate and $\alpha$-ketoglutaric semialdehyde, which is oxidized into $\alpha$-ketoglutarate by $\alpha$-ketoglutaric semialdehyde dehydrogenase [49]. All enzymes in this xylose metabolism pathway of C. crescentus are encoded by the $x y l$ operon with xylose induction [50, 51]. Additionally, 2-keto-3-deoxy-xylonate can also be split into glycolaldehyde and pyruvate by the catalysis of an aldolase in the Dahms pathway [52].

The subsequent studies have found that the native xylose metabolism pathways in some Archaea are very similar to the oxidative xylose degradation pathway in $C$. crescentus. For example, the hyperthermophilic archaeon, Sulfolobus solfataricus can metabolize glucose to glyceraldehyde and pyruvate via a non-phosphorylative variant of the Entner-Doudoroff pathway with no net ATP yield $[53,54]$. In this glucose metabolism pathway of $S$. solfataricus, the first enzyme glucose dehydrogenase and the third enzyme 2-keto-3-deoxygluconate aldolase also show the catalytic activity for pentose. Further investigation reveals that xylose can be directly converted to xylonate by glucose dehydrogenase, and then metabolized to 2-keto-3-deoxy-xylonate by a C5 specific dehydratase. Finally, the 2-keto-3-deoxy-xylonate is split into pyruvate and glycolaldehyde by the $\mathrm{C} 6$ and $\mathrm{C} 5$ promiscuity aldolase [53]. Furthermore, Johnsen et al. have reported that the xylose metabolism pathway in halophilic archaeon Haloferax volcanii shows the highly similarity to that of $C$. crescentus. Xylose is degraded to the key intermediate $\alpha$-ketoglutarate by sequentially catalytic reaction, involving the enzymes of xylose dehydrogenase (HVO_B0028), xylonolactonase (HVO_B0030), xylonate dehydratase (HVO_B0038A), 2-keto-3-deoxy-xylonate dehydratase (HVO_B0027), and $\alpha$-ketoglutarate semialdehyde dehydrogenase (HVO_B0039) [55]. The related genes in $H$. volcanii are clustered and transcriptionally regulated by a putative IclR-like regulator HVO_B0040 (designated XacR) [56]. XacR is an activator for pentose catabolism by the validation of mutant and complementation experiments. Besides that, XacR can also negatively regulate its own synthesis [56]. It is the first reported transcriptional regulator for the xylose metabolism in the domain Archaea.

In summary, xylose is converted into different intermediates and eventually metabolized completely for providing the substances and energy for cells. The xylose metabolism in different microorganisms exhibits varying degrees of difficulty but, nonetheless, more than 100 hits for xylose isomerase in the EXPASY database have been listed. It may be the important target for engineering the xylose utilization efficiency.

\section{Reconstruction of xylose metabolic pathways}

Metabolic engineering has been widely used to extend the range or improve the utilization of carbon sources in microorganisms [57]. Nowadays, xylose metabolic pathways have been reconstructed successfully in different microorganisms, such as S. cerevisiae, E. coli, Corynebacterium glutamicum, and Zymomonas mobilis [57-60]. As we know, most corynebacteria cannot utilize xylose as a carbon source because of the absence of XI. In order to bring xylose into its substrate utilization range, two recombinant $C$. glutamicum strains were constructed by cloning the E. coli XI gene xylA either alone or in combination with the $E$. coli xylulose kinase gene $x y l B$. Both engineered strains could grow in a mineral medium with xylose as the sole carbon source, and the co-expression strain grew faster on xylose compared with the strain carrying $x y l A$ alone. Moreover, the resultant strains could simultaneously consume xylose and glucose, demonstrating the absence of diauxic phenomena [59]. These results suggest that the reconstruction of the xylose metabolic pathway in microorganisms that do not utilize xylose naturally could be a useful strategy to derepress glucose inhibition.

Furthermore, the introduction of heterologous xylose metabolic genes can also improve the xylose utilization efficiency of microorganisms that catabolize xylose naturally. Two industrial S. cerevisiae strains, NAN-127 and NAN-123, were generated from the parent strain NAN27 via transforming heterologous xylose-utilizing genes. Both engineered strains showed an enhanced xylose assimilation efficiency. The xylose consumption of strain NAN-127 containing XR and $\mathrm{XDH}$ enzymes from $P$. stipitis was two times higher compared with the parent strain, with a $39 \%$ improvement in ethanol production. The xylose consumption of strain NAN-123 containing XylB enzyme from S. cerevisiae improved by $10 \%$ and the ethanol production also improved by $10 \%$ compared with that of the parent strain [58].

\section{Engineering to improve xylose uptake and utilization}

Though many microorganisms can naturally metabolize xylose, attempts for improving xylose uptake and utilization were mainly occurred in E. coli and S. cerevisiae, the most widely used hosts for microbial synthesis. In recent years, there have been rapid developments in metabolic engineering and synthetic biology, allowing the improvement of the uptake and utilization of xylose by engineering the related microorganisms, pathways, and proteins.

\section{Engineering of $x y l o s e$ transporters}

As is known, glucose can competitively inhibit all the previously reported xylose transporters, primarily 
preventing the simultaneous consumption of xylose and glucose in lignocellulose hydrolysate by microorganisms. Although XylE of E. coli is selective for xylose and does not transport glucose, xylose transport is still competitively inhibited by glucose because of the similar high affinities of glucose and xylose to XylE [61]. Much effort has been devoted to the derepression of glucose inhibition, and directed evolution of transporters has proven to be a useful method.

Directed evolution of two transporters, XUT3 from Scheffersomyces stipitis and GXS1 from C. intermedia, was conducted using yeast growth-based screening, and some mutants with improved properties were obtained. When expressed in S. cerevisiae, the mutant transporter improved the cell growth rate on xylose by up to $70 \%$ and provided the host strain with a simultaneous sugar utilization capacity in mixed glucose and xylose fermentation [8]. Similarly, the yeast hexose transporters Gal2 and Hxt7 and the $N$. crassa xylose transporter AN25 were engineered via directed evolution to transport xylose efficiently without inhibition by glucose $[42,61]$.

In directed evolution, a series of mutations at two conserved amino acid residues, threonine 219/213 and asparagine 376/370 in Hxt7 and Gal2, respectively, were identified to abolish glucose inhibition. Among these mutants, Gal2-N376F lost the ability to transport hexoses and showed the highest affinity for xylose [61]. These conserved positions were also found in XylE and other xylose transporters. A model of Gal2 was deduced from the XylE structure, showing that both residues are positioned in the central substrate-binding pocket, although neither directly binds xylose or glucose. N376F mutation impaired hexose transport completely probably because of the larger side chain protruding into the substrate translocation pathway in addition to the changed van der Waals interactions between neighboring amino acids that could alter the structure of the substrate-binding site [61]. Threonine 219/213 is indispensable for glucose transport and may play an important role in binding the C6-hydroxymethyl of glucose [62]. The analysis of conserved amino acids also suggests that N360 in Mgt05196p of Meyerozyma guilliermondii is an essential residue for xylose transport [41].

Furthermore, sugar transporter preference and kinetics can be rewired through the programming of a conserved sequence motif G-G/F-XXX-G (X represents a variable but usually nonpolar amino acid residue) in the first transmembrane span. This motif is highly conserved among functional sugar transporters. After saturation mutagenesis and subsequent rational mutagenesis, several hexose transporters acquire a high affinity for xylose, which can enable them to grow on xylose but not on glucose when expressed in a $S$. cerevisiae strain lacking endogenous monosaccharide transporters. The GGFIMG motif was found to have the highest specificity and efficiency for xylose probably because the larger side chains physically restrict the size of the pore, making it easier for smaller xylose molecules to bind and traverse compared with larger hexoses [9]. This study helps increase the understanding of the structure-function relationships of sugar transporters, providing novel insights into alleviating the effect of CCR, and it establishes a platform for engineering a specific and efficient xylose transporter.

All these results greatly promoted the construction of engineered strains that can simultaneously consume glucose and xylose. For instance, Gal2-N376F was introduced into Kluyveromyces marxianus, and the resultant strain produced ethanol and xylitol with a yield of $0.42 \mathrm{~g}$ ethanol/g glucose and $0.99 \mathrm{~g}$ xylitol/g xylose, respectively, in the co-fermentation of glucose and xylose [63].

\section{Recycling of cofactors between XR and XDH}

In fungi, xylose catabolism begins with its conversion into xylulose by XR and XDH (Fig. 1). However, there is a difference in the cofactor specificity of XR (preferential with $\mathrm{NADPH}$ ) and $\mathrm{XDH}$ (strictly with $\mathrm{NAD}^{+}$), resulting in a limited supply of $\mathrm{NAD}^{+}$for $\mathrm{XDH}$, accumulation of xylitol, and reduced production of fuels or chemicals [64]. Altering the coenzyme specificity and realizing the effective recycling of cofactors between XR and XDH have been widely performed for the improvement of the xylose fermentation efficiency.

XR encoded by the C. parapsilosis XYL1 gene is the first reported XR to prefer NADH [65]; it carries an arginine instead of a lysine in the IPKS motif conserved in NADPH-dependent XRs [66]. In order to mimic the $C$. parapsilosis $\mathrm{XR}$, a $\mathrm{K} 270 \mathrm{R}$ mutant of $P$. stipitis $\mathrm{XR}$ was made and expressed in a recombinant $S$. cerevisiae strain. In continuous fermentation, the strain carrying the $P$. stipitis XR K270 mutant gave a xylitol yield of $0.05 \mathrm{~g} / \mathrm{g}$ xylose, whereas the strain with wild-type $P$. stipitis XR gave a xylitol yield of $0.24 \mathrm{~g} / \mathrm{g}$ xylose [67]. The coenzyme specificity of $P$. stipitis XR has also been altered successfully by a semirational approach. Based on a homolog model deduced from the $C$. tenuis XR structure, six residues in $P$. stipitis $\mathrm{XR}$ were predicted to interact with the adenine ribose of $\mathrm{NAD}(\mathrm{P}) \mathrm{H}$ and become altered by saturation mutagenesis. The best mutant, carrying four mutations-K270S, S271G, N272P, and R276F-showed 13-fold improved preference for NADH compared with $\mathrm{NADPH}$. In contrast, the cofactor specificity of XDH can be completely reversed to $\mathrm{NADP}^{+}$. Four amino acid residues in P. stipitis XDH-Asp207, Ile208, Phe209, and Asn211-were selected for site-directed mutagenesis to examine their roles in the discrimination between $\mathrm{NAD}^{+}$ and $\mathrm{NADP}^{+}$. Finally, the quadruple mutant (D207A/ 
I208R/F209S/N211R) presented a $k_{\text {cat }} / K_{\mathrm{m}}$ value with $\mathrm{NADP}^{+} 4500$-fold higher compared with the wild-type enzyme, reaching a value comparable to $k_{\text {cat }} / K_{\mathrm{m}}$ with $\mathrm{NAD}^{+}$of the wild-type protein. As the thermostability of the $\mathrm{NADP}^{+}$-dependent $\mathrm{XDH}$ mutant decreased, an additional zinc-binding site composed of three cysteine residues was introduced into the quadruple mutant to improve the protein thermostability, which further increased the catalytic activity with $\mathrm{NADP}^{+}$fourfold [68]. The quadruple mutant was introduced into recombinant S. cerevisiae along with $P$. stipitis XR, and $86 \%$ decreased unfavorable xylitol accumulation and $41 \%$ increased ethanol production was found compared to the control strain carrying the wild-type XDH [69].

Evolutionary engineering for improving xylose metabolism In order to obtain the xylose-utilizing strains, a series of metabolic engineering modifications have been performed. However, it was found that the xyloseutilizing pathway is obviously affected by the different backgrounds of strains and growth conditions [70], complicating the integration of knowledge from various researches for rational design and construction. Evolutionary engineering can globally modulate metabolic networks without extensive regulatory or genetic information about the desirable phenotypes, appropriate for improving the utilization of unfavorable carbon sources.

Escherichia coli MG1655 was engineered to produce D-lactate from xylose by the deletion of the $p f l B, a d h E$, frdA, and $x y l F G H$ genes [71]. Knockout of $p f l B, a d h E$ and frdA, involved in production of by-product formate, ethanol and succinate, may improve the xylose conversion efficiency. The $x y l F G H$ genes encoding ATP-dependent xylose transporter was deleted to increase ATP availability. In order to further improve cell growth and lactate productivity, this strain was applied to adaptive evolution on xylose. The evolved strain showed a 50\% increase in the growth rate and lactate productivity and a twofold increase in the xylose consumption rate. Whole-genome sequencing revealed a point mutation in the GatC protein, which resulted in the replacement of serine by leucine at position 184. This modified GatC protein can be used as a new xylose transporter that improves the growth and consumption rate of xylose in that evolved strain [71].

A recombinant $S$. cerevisiae strain KF7M-16, carrying the $P$. stipitis XR-XDH pathway, was also subjected to adaptive evolution. The evolved strain YNBX26-21 showed significantly improved cell growth and ethanol production rate. Comparative transcriptome analysis revealed novel responses to xylose, including the biosynthesis of vitamins B1 and B6 and sulfur amino acid and decreased expression of $\mathrm{Fe}(\mathrm{II})$ transport-related genes and several glucose-repressible genes, which probably contributed to the improved xylose utilization [72]. All these results from adaptive evolution provided new insights into the construction of a superior xylose-utilizing strain through inverse metabolic engineering.

\section{Biosynthesis of chemicals from xylose}

In recent years, the bioconversion of xylose into valueadded chemicals has attracted considerable attention. Many wild microbial strains or engineered strains have been identified or constructed to utilize xylose for producing a variety of compounds, including ethanol, 1,4-butanediol (BDO), 1,2,4-butanetriol (BT), ethylene glycol (EG), glycolate, xylitol [29, 63], and succinate [73, 74] (Table 1).

\section{Ethanol}

Ethanol is a promising biochemical that is widely used as a gasoline additive and biofuel. In recent years, $S$. cerevisiae have been widely used for ethanol production because they are highly tolerant to ethanol [75]. Besides the strategies of xylose-utilizing pathway reconstruction, redox balancing, and adaptive evolution described above, an efficient, simple, and programmable approach for the rapid tuning of gene expression in a heterologous pathway, named "customized optimization of metabolic pathways by combinatorial transcriptional engineering (COMPACTER)", was used to improve ethanol productivity from xylose in recombinant $S$. cerevisiae carrying the xylose reductase from Candida shehatae (csXR), the xylitol dehydrogenase from Candida tropicalis (ctXDH) and the xylulose kinase from Pichia pastoris (ppXKS) [70]. Specifically, each gene in the xylose-utilizing pathway was assembled with promoter mutants of varying strengths to construct a library of mutant pathways, which was subjected to high-throughput screening. After a single round of COMPACTER, the optimized mutant produced ethanol 1.5 times faster and consumed xylose $70 \%$ faster compared with the reference strain carrying the same xylose metabolic pathway under the control of wild-type promoters.

Besides S. cerevisiae systems, other microorganisms were also developed to produce ethanol from xylose, such as E. coli, Z. mobilis, and P. stipitis. Among these, $Z$. mobilis is one of the best ethanol producers found in nature owing to their high theoretical ethanol yield from sugars and high ethanol productivity rate [76]. However, Z. mobilis do not have the native xylose metabolism pathway. In order to expand their substrate range to xylose, two operons encoding E. coli XylAB and PPP enzymes were cloned and transformed into Z. mobilis, conferring the co-fermentation of glucose and xylose to ethanol [60]. To further improve xylose fermentation, 
Table 1 Metabolism of xylose in different recombinant microorganisms for high-value chemicals and biofuels production

\begin{tabular}{|c|c|c|c|c|c|c|}
\hline Strain & Substrate & Chemicals & Titer (g/L) & Yield (g/g)-xylose & Productivity (g/L/h) & References \\
\hline Z. mobilis & Xylose & Ethanol & 43.1 & 0.45 & 0.94 & [77] \\
\hline S. cerevisiae & Xylose & Ethanol & 16.4 & 0.41 & 0.77 & [89] \\
\hline S. cerevisiae & Xylose & Ethanol & 10.8 & 0.25 & 0.39 & [90] \\
\hline S. cerevisiae & Xylose & Ethanol & 10.4 & 0.26 & 0.24 & [70] \\
\hline E. coli & Xylose & BT & 5.1 & 0.255 & 0.106 & [81] \\
\hline E. coli & Xylose & BT & 3.92 & 0.20 & 0.05 & [78] \\
\hline E. coli & Xylonate & BT & 1.6 & 0.25 & & {$[80]$} \\
\hline E. coli & Xylose & BT & 0.88 & 0.13 & 0.012 & [91] \\
\hline E. coli & Xylose & $\mathrm{BDO}$ & 0.209 & 0.01 & 0.004 & {$[82]$} \\
\hline E. coli & Xylose & EG & 108.2 & 0.36 & 2.25 & {$[88]$} \\
\hline E. coli & Xylose & EG & 72 & 0.40 & 1.38 & [87] \\
\hline E. coli & Xylose & EG & 40 & 0.35 & 0.56 & [83] \\
\hline E. coli & xylose & EG & 20 & 0.38 & 0.37 & {$[86]$} \\
\hline E. coli & Xylose & Glycolate & 44 & 0.44 & 0.92 & [83] \\
\hline E. coli & Xylose & Glycolate & 43.6 & 0.46 & 0.91 & [84] \\
\hline K. lactis & $\begin{array}{l}\text { Xylose } \\
\text { Ethanol }\end{array}$ & Glycolate & 14.8 & 0.28 & 0.09 & [92] \\
\hline E. coli & Xylose & Glycolate & 4.57 & 0.46 & - & [93] \\
\hline E. coli & Xylose & Glycolate & 4.3 & 0.46 & 0.089 & [94] \\
\hline
\end{tabular}

adaptive evolution involving 30 transfers in a medium containing a high concentration of xylose was conducted, and a strain A3 finally obtained the significant improvement of xylose metabolism. This strain was able to grow on $100 \mathrm{~g} / \mathrm{L}$ xylose and rapidly fermented xylose to ethanol within 2 days. The final titer of ethanol was $43.1 \mathrm{~g} / \mathrm{L}$ with a yield of $0.45 \mathrm{~g} / \mathrm{g}$ xylose and ethanol productivity of $0.94 \mathrm{~g} / \mathrm{L} / \mathrm{h}$ [77]. These results revealed that metabolic engineering and adaptive evolution could be used synergistically for strain improvement.

\section{BT and BDO}

BT is an important fine chemical and has versatile applications in many fields. For example, it can be used for making polyurethane foam, which has compressionbending properties similar to natural rubber. As a potential building block, BT is also used for synthesizing various pharmaceuticals and is used as a direct precursor for BT trinitrate, a great energetic plasticizer [78]. BDO is a valuable commodity chemical, with an annual market of an excess of 2.5 million tons worldwide. As a chemical intermediate, $\mathrm{BDO}$ goes into an extraordinarily wide spectrum of products, including automotive parts, electronics, and apparel [79].

The first report on the biosynthesis of BT employed two microbial strains. Xylose is initially converted to xylonate by Pseudomonas fragi, and the E. coli construct is responsible for the conversion of xylonate into $\mathrm{BT}$, which carries a 2-keto acid decarboxylase gene
mdlC from Pseudomonas putida (Fig. 2; [80]. However, the production and purification of xylonate is a tedious process that increases the whole production cost of BT. Thus, a recombinant $E$. coli strain was constructed to directly convert xylose into BT [78]. The $\mathrm{Xdh}$ XylB and XylC from Caulobacter crescentus and 2-keto acid decarboxylase MdlC from P. putida were co-expressed with the native xylonate dehydratase YjhG and aldehyde reductase AdhP in E. coli BL21 star (DE3) (Fig. 2). Meanwhile, the endogenous XI XylA and xylulose kinase XylB were deleted to block the native xylose metabolism pathway. The final constructed strain produced $3.92 \mathrm{~g} / \mathrm{L} \mathrm{BT}$ from $20 \mathrm{~g} / \mathrm{L}$ xylose, corresponding to a yield of $0.20 \mathrm{~g} / \mathrm{g}$ xylose. A similar BT-producing strain was constructed using aldehyde reductase YqhD instead of AdhP. In combination with the knockout of 2-keto acid reductase YiaE to eliminate the competing branch pathway, this strain produced $5.1 \mathrm{~g} / \mathrm{L} \mathrm{BT}$ with a yield of $0.26 \mathrm{~g} / \mathrm{g}$ xylose [81].

Based on the BT-producing route, a de novo biosynthetic pathway of BDO was established (Fig. 2; [82]. The Klebsiella oxytoca diol dehydratase, which dehydrates its native substrate 1,2-propanediol efficiently, was rationally engineered to promote catalysis toward a nonnative triol, BT. The mutant enzyme showed dehydratase activity toward BT by nearly fivefold in comparison to the wild-type protein. By introducing the complete pathway into the $E$. coli strain, the biosynthesis of BDO at $209 \mathrm{mg} / \mathrm{L}$ from xylose was achieved. 


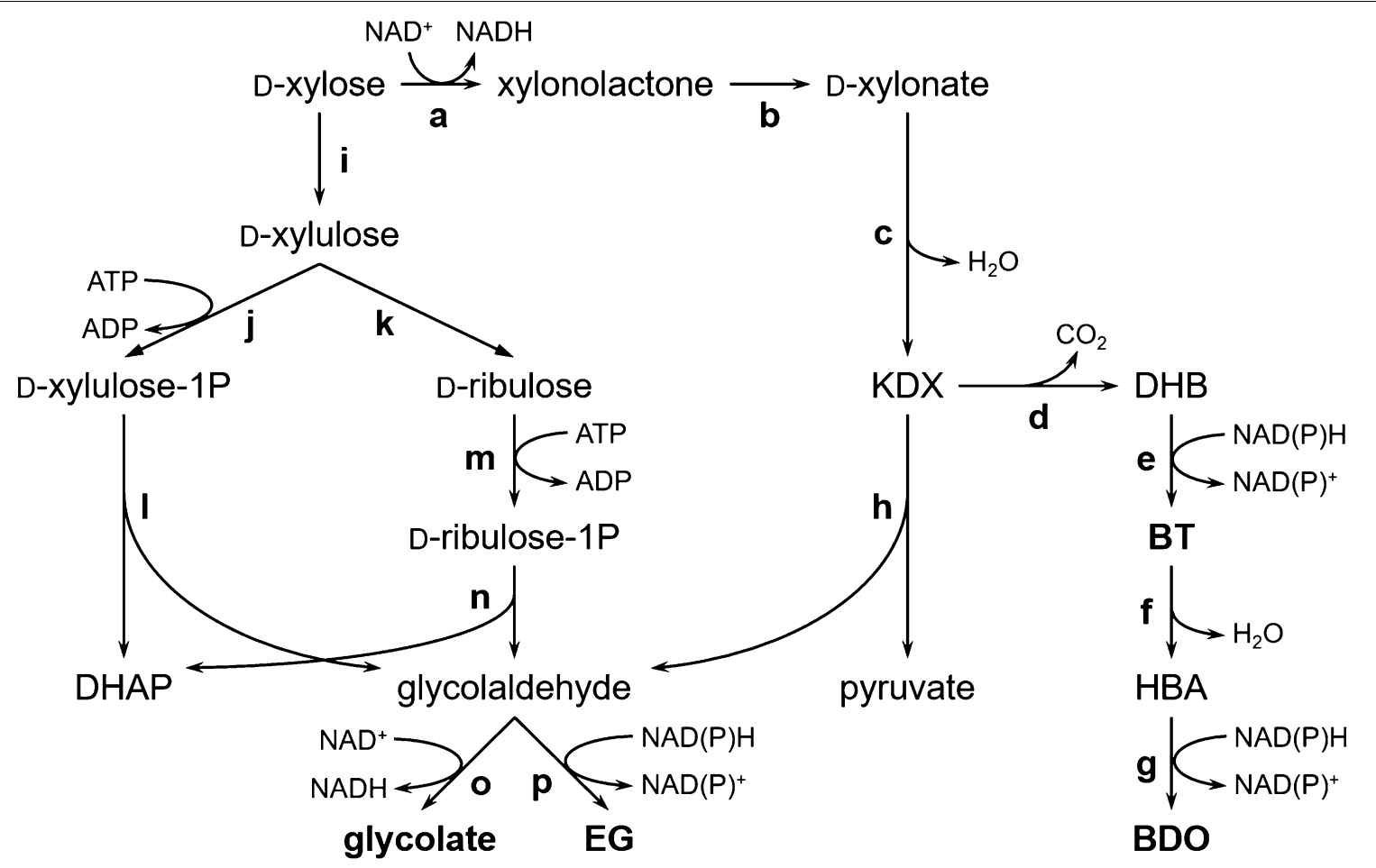

Fig. 2 Biosynthetic pathways of chemicals from xylose. DHAP, dihydroxyacetone phosphate; EG, ethylene glycol; KDX, 2-keto-3-deoxy-D-xylonate; DHB, D-3,4-dihydroxybutanal; BT, D-1,2,4-butanetriol; HBA, 4-hydroxybutyraldehyde; BDO, 1,4-butanediol. a D-xylose dehydrogenase, XlyB from C. crescentus; b xylonolactonase, XylC from C. Crescentus; C D-xylonate dehydratase, YjhG/YagF from E. coli, XyID from C. crescentus; d decarboxylase, KivD from Lactococcus lactis, MdIC from Pseudomonas putida; e alcohol dehydrogenase, YqhD/AdhP from E. coli, ADH2 from S. cerevisiae; f diol dehydratase, engineered PpdA-C-B fusion from Klebsiella oxytoca; g alcohol dehydrogenase, YqhD from E. coli; h 2-keto-3-deoxy-D-pentanoate aldolase, YjhH/YagE from E. coli; i D-xylose isomerase, XyIA from E. coli; j D-xylulose-1-kinase, Khk-C from human; k d-tagatose 3-epimerase, DTE from Pseudomonas cichorii; I D-xylulose-1-phosphate aldolase, Aldo-B from human; $\mathrm{m}$ L-fuculokinase, Fuck from E. coli; $\mathrm{n}$ L-fuculose-1-phosphate aldolase, FucA from E. coli; o aldehyde dehydrogenase, AldA from E. coli; p alcohol dehydrogenase, FucO/YqhD from E. coli

\section{EG and glycolate}

EG is a commodity chemical that can be used in multiple everyday applications. There are two most prominent uses of EG: automotive antifreeze and one of the precursors for poly(ethylene terephthalate). Moreover, EG can be converted into acetaldehyde with the help of diol dehydratase and then transformed into ethanol, acetate, or acetyl-CoA [83]. Glycolate is an organic acid containing both carboxyl and alcohol groups. It is an ideal choice for a wide range of applications, such as a precursor for biopolymer synthesis, a rinsing agent in the tanning and dyeing industry, and a skincare product in the cosmetics industry. Additionally, the glycolate polymer is a new type of packaging material with excellent performance because of its gas-barrier and mechanical properties [84]. With a growing demand, the global market of glycolate is expected to reach USD 277.8 million in 2020 (http:// www.grandviewresearch.com/press-release/-glycolicacid-market).

The biosynthetic pathways for EG and glycolate were successfully reconstructed in E. coli. They are both derived from the intermediate glycolaldehyde, which can be produced from xylose via three different routes (Fig. 2). In the Dahms pathway, the metabolite 2-keto3-deoxy-xylonate can be split into glycolaldehyde and pyruvate by $E$. coli endogenous pentanoate aldolase $\mathrm{YjhH} / \mathrm{YagE}[84,85]$. In contrast, xylose is initially isomerized to xylulose, which is further transformed into xylulose-1-phosphate or ribulose-1-phosphate in different recombinant strains $[83,86]$. Then, pentose-1-phosphate is split into glycolaldehyde and dihydroxyacetone phosphate by Aldo-B from human or FucA from E. coli (Fig. 2). The conversion of xylose into each mole of glycolaldehyde via the Dahms pathway generates one mole of NADH, whereas one mole of ATP is consumed in both routes with xylulose-1-phosphate and ribulose-1-phosphate as intermediates.

In order to improve the productivity of EG and glycolate, strategies including redox balancing [87], elimination of competitive branch pathways [86], and overcoming acetate overflow $[84,87]$ were performed. Up till now, the highest titers of EG and 
glycolate produced from xylose are $108 \mathrm{~g} / \mathrm{L}$ with a yield of $0.36 \mathrm{~g} / \mathrm{g}$ xylose [88] and $44 \mathrm{~g} / \mathrm{L}$ with a yield of $0.44 \mathrm{~g} / \mathrm{g}$ xylose [83], respectively.

In summary, all these results fully confirm the great potential of xylose as a carbon source for the biosynthesis of high-value chemicals and biofuel. It is useful to extensively use xylose to fully explore lignocellulose resources and provide a new direction for microbial fermentation.

\section{Conclusion}

As the second most abundant sugar in lignocellulose, xylose is a promising renewable resource for producing biofuels and chemicals, and much effort has been expended in xylose bioconversion. A number of xylose transporters from E. coli and filamentous fungi were discovered and characterized, and five xylose assimilation pathways were identified in various microorganisms. In microorganisms that cannot metabolize xylose naturally, such as S. cerevisiae, artificial pathways were reconstructed by the introduction of heterologous xylose transporter and catabolic genes. Value-added chemicals, including ethanol, BT, BDO, EG, and glycolate, were synthesized from xylose by recombinant microorganisms, and the productions were significantly improved through metabolic engineering. Despite the achievements that have been made, there are still some bottlenecks in xylose bioconversion. First, glucose represses the activity of xylose transporters as well as the transcription of xylose-related genes, hampering the co-utilization of glucose and xylose in lignocellulose hydrolysate. It is necessary to focus on further developments in order to explore new xylose transporters without glucose inhibition and establish cAMP-CRP-independent transcriptional regulation for genes involved in xylose utilization. Second, the conversion efficiency of xylose to target chemicals is low, resulting in a high production cost. We believe that the yield should increase significantly in the future, benefiting from the development of new technologies in enzyme discovery and optimization, fine-tuning of the expression level of multiple genes in a complex pathway, and evolutionary engineering, among other methods.

\footnotetext{
Abbreviations

S. cerevisiae: Saccharomyces cerevisiae; E. coli: Escherichia coli; A. nidulans: Aspergillus nidulans; Z. mobilis: Zymomonas mobilis; CCR: Carbon catabolite repression; ABC: ATP-binding cassette; MFS: The major facilitator superfamily; PTS: Carbohydrate Phosphotransferase System; XI: Xylose isomerase; XR: Xylose reductase; XDH: Xylitol dehydrogenase; PPP: Pentose phosphate pathway; PK: Phosphoketolase; BDO: 1,4-Butanediol; BT: 1,2,4-Butanetriol; EG: Ethylene glycol.
}

Acknowledgements

Not applicable.

\section{Authors' contributions}

MX and GZ developed the idea for the review. ZZ, ML and GZ participated in the literature research and paper writing. $M X$ helped to revise this manuscript. All authors read and approved the final manuscript.

\section{Funding}

This work was supported by the National Natural Science Foundation of China (31722001 and 21807101), the Natural Science Foundation of Shandong province (JQ201707), Defense Industrial Technology Development Program (JCKY2018130B005), CAS Key Program (KJZD-EW-G20).

Availability of data and materials

Not applicable.

Ethics approval and consent to participate

Not applicable.

Consent for publication

All authors read and approved the final manuscript for publication.

\section{Competing interests}

The authors declare that they have no competing interests.

\section{Author details}

${ }^{1}$ CAS Key Laboratory of Biobased Materials, Qingdao Institute of Bioenergy and Bioprocess Technology, Chinese Academy of Sciences, Qingdao 266101, China. ${ }^{2}$ University of Chinese Academy of Sciences, Beijing 100049, China.

Received: 15 October 2019 Accepted: 21 January 2020

Published online: 01 February 2020

\section{References}

1. Kim JS, Lee Y, Kim TH. A review on alkaline pretreatment technology for bioconversion of lignocellulosic biomass. Bioresour Technol. 2016;199:42-8.

2. Hahn-Hägerdal B, Karhumaa K, Fonseca C, Spencer-Martins I, GorwaGrauslund MF. Towards industrial pentose-fermenting yeast strains. Appl Microbiol Biotechnol. 2007;74:937-53.

3. de Paula RG, Antonieto ACC, Ribeiro LFC, Srivastava N, O'Donovan A, Mishra PK, Gupta VK, Silva RN. Engineered microbial host selection for value-added bioproducts from lignocellulose. Biotechnol Adv. 2019. https://doi.org/10.1016/j.biotechadv.2019.02.003.

4. Van Dyk JS, Pletschke BI. A review of lignocellulose bioconversion using enzymatic hydrolysis and synergistic cooperation between enzymesfactors affecting enzymes, conversion and synergy. Biotechnol Adv. 2012:30:1458-80.

5. Buijs NA, Siewers V, Nielsen J. Advanced biofuel production by the yeast Saccharomyces cerevisiae. Curr Opin Chem Biol. 2013;17:480-8.

6. Park JM, Vinuselvi P, Lee SK. The mechanism of sugar-mediated catabolite repression of the propionate catabolic genes in Escherichia coli. Gene. 2012;504:116-21.

7. Parachin NS, Bergdahl B, van Niel EW, Gorwa-Grauslund MF. Kinetic modelling reveals current limitations in the production of ethanol from xylose by recombinant Saccharomyces cerevisiae. Metab Eng. 2011;13:508-17.

8. Young EM, Comer AD, Huang H, Alper HS. A molecular transporter engineering approach to improving xylose catabolism in Saccharomyces cerevisiae. Metab Eng. 2012;14:401-11.

9. Young EM, Tong A, Bui H, Spofford C, Alper HS. Rewiring yeast sugar transporter preference through modifying a conserved protein motif. Proc Natl Acad Sci USA. 2014;111:131-6.

10. Tanimura K, Matsumoto T, Nakayama H, Tanaka T, Kondo A. Improvement of ectoine productivity by using sugar transporter-overexpressing Halomonas elongata. Enzyme Microb Technol. 2016;89:63-8.

11. Pao SS, Paulsen IT, Saier MH Jr. Major facilitator superfamily. Microbiol Mol Biol Rev. 1998;62:1-34.

12. Yan N. Structural advances for the major facilitator superfamily (MFS) transporters. Trends Biochem Sci. 2013;38:151-9.

13. Khankal R, Chin JW, Cirino PC. Role of xylose transporters in xylitol production from engineered Escherichia coli. J Biotechnol. 2008;134:246-52. 
14. Hasona A, Kim Y, Healy FG, Ingram LO, Shanmugam KT. Pyruvate formate lyase and acetate kinase are essential for anaerobic growth of Escherichia coli on xylose. J Bacteriol. 2004;186:7593-600.

15. Sumiya M, Davis EO, Packman LC, McDonald TP, Henderson PJ. Molecular genetics of a receptor protein for $D$-xylose, encoded by the gene $x y / F$, in Escherichia coli. Recept Channels. 1995;3:117-28.

16. Sun L, Zeng X, Yan C, Sun X, Gong X, Rao Y, Yan N. Crystal structure of a bacterial homologue of glucose transporters GLUT1-4. Nature. 2012:490:361-6.

17. Sofia HJ, Burland V, Daniels DL, Plunkett G 3rd, Blattner FR. Analysis of the Escherichia coli genome $V$ DNA sequence of the region from 76.0 to 81.5 minutes. Nucleic Acids Res. 1994;22:2576-86.

18. Ahlem C, Huisman W, Neslund G, Dahms AS. Purification and properties of a periplasmic D-xylose-binding protein from Escherichia coli K-12. J Biol Chem. 1982;257:2926-31.

19. Jones DT, Woods DR. Acetone-butanol fermentation revisited. Microb Rev. 1986;50:484-524

20. Gu Y, Ding Y, Ren C, Sun Z, Rodionov DA, Zhang W, Yang S, Yang C, Jiang W. Reconstruction of xylose utilization pathway and regulons in Firmicutes. BMC Genomics. 2010;11:255.

21. Gu Y, Jiang Y, Yang S, Jiang W. Utilization of economical substrate-derived carbohydrates by solventogenic clostridia: pathway dissection, regulation and engineering. Curr Opin Biotechnol. 2014;29:124-31.

22. Wagner M, Shen $L$, Albersmeier A, van der Kolk N, Kim S, Cha J, Bräsen C, Kalinowski J, Siebers B, Albers S-V. Sulfolobus acidocaldarius transports pentoses via a carbohydrate uptake transporter 2 (CUT2)-type ABC transporter and metabolizes them through the aldolase-independent Weimberg pathway. Appl Environ Microbiol. 2018;84:e01217-73.

23. Elferink MG, Albers SV, Konings WN, Driessen AJ. Sugar transport in Sulfolobus solfataricus is mediated by two families of binding proteindependent ABC transporters. Mol Microbiol. 2001:39:1494-503.

24. Choi K-H, Hwang S, Cha J. Identification and characterization of MalA in the maltose/maltodextrin operon of Sulfolobus acidocaldarius DSM639. J Bacteriol. 2013;195:1789-99.

25. Song S, Park C. Organization and regulation of the D-xylose operons in Escherichia coli K-12: XyIR acts as a transcriptional activator. J Bacteriol. 1997;179:7025-32.

26. Kim JH, Block DE, Mills DA. Simultaneous consumption of pentose and hexose sugars: an optimal microbial phenotype for efficient fermentation of lignocellulosic biomass. Appl Microbiol Biotechnol. 2010;88:1077-85.

27. Gorke B, Stulke J. Carbon catabolite repression in bacteria: many ways to make the most out of nutrients. Nat Rev Microbiol. 2008:6:613-24.

28. Karimova G, Ladant D, Ullmann A. Relief of catabolite repression in a CAMP-independent catabolite gene activator mutant of Escherichia coli. Res Microbiol. 2004;155:76-9.

29. Cirino PC, Chin JW, Ingram LO. Engineering Escherichia coli for xylitol production from glucose-xylose mixtures. Biotechnol Bioeng. 2006;95:1167-76.

30. Khankal R, Luziatelli F, Chin JW, Frei CS, Cirino PC. Comparison between Escherichia coli K-12 strains W3110 and MG1655 and wild-type E. coli B as platforms for xylitol production. Biotechnol Lett. 2008;30:1645-53.

31. Gonzalez R, Tao H, Shanmugam KT, York SW, Ingram LO. Global gene expression differences associated with changes in glycolytic flux and growth rate in Escherichia coli during the fermentation of glucose and xylose. Biotechnol Prog. 2002;18:6-20.

32. Xiao $H$, Li Z, Jiang Y, Yang Y, Jiang W, Gu Y, Yang S. Metabolic engineering of D-xylose pathway in Clostridium beijerinckii to optimize solvent production from xylose mother liquid. Meta Eng. 2012;14:569-78.

33. Warner JB, Lolkema JS. CcpA-dependent carbon catabolite repression in bacteria. Microbiol Mol Biol Rev. 2003;67:475-90.

34. Du J, Li S, Zhao H. Discovery and characterization of novel D-xylose-specific transporters from Neurospora crassa and Pichia stipitis. Mol Biosyst. 2010;6:2150-6.

35. Saloheimo A, Rauta J, Stasyk OV, Sibirny AA, Penttila M, Ruohonen L. Xylose transport studies with xylose-utilizing Saccharomyces cerevisiae strains expressing heterologous and homologous permeases. Appl Microbiol Biotechnol. 2007;74:1041-52.

36. Colabardini AC, Ries LN, Brown NA, Dos Reis TF, Savoldi M, Goldman $\mathrm{MH}$, Menino JF, Rodrigues F, Goldman GH. Functional characterization of a xylose transporter in Aspergillus nidulans. Biotechnol Biofuels. 2014:7:46-65.
37. Ferreira ME, Colombo AL, Paulsen I, Ren Q, Wortman J, Huang J, Goldman $\mathrm{MH}$, Goldman $\mathrm{GH}$. The ergosterol biosynthesis pathway, transporter genes, and azole resistance in Aspergillus fumigatus. Med Mycol. 2005;43(Suppl 1):S313-9.

38. Dos Reis TF, de Lima PB, Parachin NS, Mingossi FB, de Castro Oliveira JV, Ries LN, Goldman GH. Identification and characterization of putative xylose and cellobiose transporters in Aspergillus nidulans. Biotechnol Biofuels. 2016;9:204-23.

39. Ries L, Pullan ST, Delmas S, Malla S, Blythe MJ, Archer DB. Genome-wide transcriptional response of Trichoderma reesei to lignocellulose using RNA sequencing and comparison with Aspergillus niger. BMC Genomics. 2013;14:541-53.

40. Vankuyk PA, Diderich JA, MacCabe AP, Hererro O, Ruijter GJ, Visser J. Aspergillus niger mstA encodes a high-affinity sugar $/ \mathrm{H}^{+}$symporter which is regulated in response to extracellular pH. Biochem J. 2004;379:375-83.

41. Wang C, Bao X, Li Y, Jiao C, Hou J, Zhang Q, Zhang W, Liu W, Shen Y. Data set for cloning and characterization of heterologous transporters in Saccharomyces cerevisiae and identification of important amino acids for xylose utilization. Data Brief. 2015;4:119-26.

42. Wang $M$, Yu C, Zhao H. Directed evolution of xylose specific transporters to facilitate glucose-xylose co-utilization. Biotechnol Bioeng. 2016;113:484-91.

43. Jeffries TW. Utilization of xylose by bacteria, yeasts, and fungi. In: Pentoses and Lignin. Berlin: Springer; 1983: p. 1-32.

44. Mishra P, Singh A. Microbial pentose utilization. Adv Appl Microbiol. 1993;39:91-152.

45. Wang PY, Shopsis C, Schneider H. Fermentation of a pentose by yeasts. Biochem Biophys Res Commun. 1980;94:248-54

46. Scalcinati G, Otero JM, Van Vleet JR, Jeffries TW, Olsson L, Nielsen J. Evolutionary engineering of Saccharomyces cerevisiae for efficient aerobic xylose consumption. FEMS Yeast Res. 2012;12:582-97.

47. Liu L, Zhang L, Tang W, Gu Y, Hua Q, Yang S, Jiang W, Yang C. Phosphoketolase pathway for xylose catabolism in Clostridium acetobutylicum revealed by ${ }^{13} \mathrm{C}$ metabolic flux analysis. J Bacteriol. 2012;194:5413-22.

48. Tanaka K, Komiyama A, Sonomoto K, Ishizaki A, Hall SJ, Stanbury PF. Two different pathways for D-xylose metabolism and the effect of xylose concentration on the yield coefficient of L-lactate in mixed-acid fermentation by the lactic acid bacterium Lactococcus lactis IO-1. Appl Microbiol Biotechnol. 2002;60:160-7.

49. Stephens C, Christen B, Fuchs T, Sundaram V, Watanabe K, Jenal U. Genetic analysis of a novel pathway for D-xylose metabolism in Caulobacter crescentus. J Bacteriol. 2007;189:2181-5.

50. Stephens C, Christen B, Watanabe K, Fuchs T, Jenal U. Regulation of D-xylose metabolism in Caulobacter crescentus by a Lacl-type repressor. J Bacteriol. 2007;189:8828-34.

51. Meisenzahl AC, Shapiro L, Jenal U. Isolation and characterization of a xylose-dependent promoter from Caulobacter crescentus. J Bacteriol. 1997;179:592-600.

52. Dahms AS. 3-Deoxy-D-pentulosonic acid aldolase and its role in a new pathway of D-xylose degradation. Biochem Biophys Res Commun. 1974;60:1433-9.

53. Nunn CE, Johnsen U, Schönheit P, Fuhrer T, Sauer U, Hough DW, Danson MJ. Metabolism of pentose sugars in the hyperthermophilic archaea Sulfolobus solfataricus and Sulfolobus acidocaldarius. J Biol Chem. 2010;285:33701-9.

54. Siebers B, Schönheit P. Unusual pathways and enzymes of central carbohydrate metabolism in Archaea. Curr Opin Microbiol. 2005;8:695-705.

55. Johnsen U, Dambeck M, Zaiss H, Fuhrer T, Soppa J, Sauer U, Schönheit P. D-xylose degradation pathway in the halophilic archaeon Haloferax volcanii. J Biol Chem. 2009:284:27290-303.

56. Johnsen U, Sutter JM, Schulz AC, Tästensen JB, Schönheit P. XacR-a novel transcriptional regulator of $D$-xylose and L-arabinose catabolism in the haloarchaeon H aloferax volcanii. Environ Microbiol. 2015;17:1663-76.

57. Young $E$, Lee $S M$, Alper $H$. Optimizing pentose utilization in yeast: the need for novel tools and approaches. Biotechnol Biofuels. 2010;3:24-36.

58. Wang Y, Shi WL, Liu XY, Shen Y, Bao XM, Bai FW, Qu YB. Establishment of a xylose metabolic pathway in an industrial strain of Saccharomyces cerevisiae. Biotechnol Lett. 2004;26:885-90.

59. Kawaguchi $H$, Vertes AA, Okino S, Inui M, Yukawa $H$. Engineering of a xylose metabolic pathway in Corynebacterium glutamicum. Appl Environ Microbiol. 2006:72:3418-28. 
60. Zhang M, Eddy C, Deanda K, Finkelstein M, Picataggio S. Metabolic engineering of a pentose metabolism pathway in Ethanologenic Zymomonas mobilis. Science. 1995;267:240-3.

61. Farwick A, Bruder S, Schadeweg V, Oreb M, Boles E. Engineering of yeast hexose transporters to transport D-xylose without inhibition by D-glucose. Proc Natl Acad Sci USA. 2014;111:5159-64.

62. Kasahara T, Shimogawara K, Kasahara M. Crucial effects of amino acid side chain length in transmembrane segment 5 on substrate affinity in yeast glucose transporter Hxt7. Biochemistry. 2011;50:8674-81.

63. Zhang B, Zhang J, Wang D, Han R, Ding R, Gao X, Sun L, Hong J. Simultaneous fermentation of glucose and xylose at elevated temperatures coproduces ethanol and xylitol through overexpression of a xylose-specific transporter in engineered Kluyveromyces marxianus. Bioresour Technol. 2016;216:227-37.

64. Ostergaard S, Olsson L, Nielsen J. Metabolic engineering of Saccharomyces cerevisiae. Microbiol Mol Biol Rev. 2000;64:34-50.

65. Lee JK, Koo BS, Kim SY. Cloning and characterization of the xyl1 gene, encoding an $\mathrm{NADH}$-preferring xylose reductase from Candida parapsilosis, and its functional expression in Candida tropicalis. Appl Environ Microbiol. 2003;69:6179-88.

66. Lee $\mathrm{H}$. The structure and function of yeast xylose (aldose) reductases. Yeast. 1998;14:977-84

67. Bengtsson O, Hahn-Hagerdal B, Gorwa-Grauslund MF. Xylose reductase from Pichia stipitis with altered coenzyme preference improves ethanolic xylose fermentation by recombinant Saccharomyces cerevisiae. Biotechnol Biofuels. 2009;2:9-19.

68. Watanabe S, Kodaki T, Makino K. Complete reversal of coenzyme specificity of xylitol dehydrogenase and increase of thermostability by the introduction of structural zinc. J Biol Chem. 2005;280:10340-9.

69. Watanabe S, Saleh AA, Pack SP, Annaluru N, Kodaki T, Makino K. Ethanol production from xylose by recombinant Saccharomyces cerevisiae expressing protein engineered $\mathrm{NADP}^{+}$-dependent xylitol dehydrogenase. J Biotechnol. 2007;130:316-9.

70. Du J, Yuan Y, Si T, Lian J, Zhao H. Customized optimization of metabolic pathways by combinatorial transcriptional engineering. Nucleic Acids Res. 2012;40:e142.

71. Utrilla J, Licona-Cassani C, Marcellin E, Gosset G, Nielsen LK, Martinez A. Engineering and adaptive evolution of Escherichia coli for D-lactate fermentation reveals GatC as a xylose transporter. Metab Eng. 2012;14:469-76.

72. Zeng WY, Tang YQ, Gou M, Sun ZY, Xia ZY, Kida K. Comparative transcriptomes reveal novel evolutionary strategies adopted by Saccharomyces cerevisiae with improved xylose utilization capability. Appl Microbiol Biotechnol. 2017;101:1753-67.

73. Liu R, Liang L, Chen K, Ma J, Jiang M, Wei P, Ouyang P. Fermentation of xylose to succinate by enhancement of ATP supply in metabolically engineered Escherichia coli. Appl Microbiol Biotechnol. 2012;94:959-68.

74. Zhang F, Li J, Liu H, Liang Q, Qi Q. ATP-Based ratio regulation of glucose and xylose improved succinate production. PLoS ONE. 2016;11:e0157775.

75. Kim SR, Park Y-C, Jin Y-S, Seo J-H. Strain engineering of Saccharomyces cerevisiae for enhanced xylose metabolism. Biotechnol Adv. 2013;31:851-61.

76. Panesar PS, Marwaha SS, Kennedy JF. Zymomonas mobilis: an alternative ethanol producer. J Chem Technol Biotechnol. 2006:81:623-35.

77. Agrawal M, Mao Z, Chen RR. Adaptation yields a highly efficient xylose-fermenting Zymomonas mobilis strain. Biotechnol Bioeng. 2011;108:777-85.

78. Cao Y, Niu W, Guo J, Xian M, Liu H. Biotechnological production of 1,2,4-butanetriol: an efficient process to synthesize energetic material precursor from renewable biomass. Sci Rep. 2015;5:18149-58.
79. Burgard A, Burk MJ, Osterhout R, Van Dien S, Yim H. Development of a commercial scale process for production of 1,4-butanediol from sugar. Curr Opin Biotechnol. 2016;42:118-25.

80. Niu W, Molefe MN, Frost JW. Microbial synthesis of the energetic material precursor 1,2,4-butanetriol. J Am Chem Soc. 2003;125:12998-9.

81. Zhang N, Wang J, Zhang Y, Gao H. Metabolic pathway optimization for biosynthesis of 1,2,4-butanetriol from xylose by engineered Escherichia coli. Enzyme Microb Technol. 2016;93-94:51-8.

82. Wang J, Jain R, Shen X, Sun X, Cheng M, Liao JC, Yuan Q, Yan Y. Rational engineering of diol dehydratase enables 1,4-butanediol biosynthesis from xylose. Metab Eng. 2017;40:148-56.

83. Pereira B, Li ZJ, De Mey M, Lim CG, Zhang H, Hoeltgen C, Stephanopoulos G. Efficient utilization of pentoses for bioproduction of the renewable two-carbon compounds ethylene glycol and glycolate. Metab Eng. 2016;34:80-7.

84. Liu M, Ding Y, Xian M, Zhao G. Metabolic engineering of a xylose pathway for biotechnological production of glycolate in Escherichia coli. Microb Cell Fact. 2018;17:51-62.

85. Liu H, Ramos KR, Valdehuesa KN, Nisola GM, Lee WK, Chung WJ. Biosynthesis of ethylene glycol in Escherichia coli. Appl Microbiol Biotechnol. 2013;97:3409-17.

86. Alkim C, Cam Y, Trichez D, Auriol C, Spina L, Vax A, Bartolo F, Besse P, Francois JM, Walther T. Optimization of ethylene glycol production from (D)-xylose via a synthetic pathway implemented in Escherichia coli. Microb Cell Fact. 2015;14:127-39.

87. Wang Y, Xian M, Feng X, Liu M, Zhao G. Biosynthesis of ethylene glycol from D-xylose in recombinant Escherichia coli. Bioengineered. 2018;9:233-41.

88. Chae TU, Choi SY, Ryu JY, Lee SY. Production of ethylene glycol from xylose by metabolically engineered Escherichia coli. AIChE J. 2018;64:4193-200.

89. Zhou H, Cheng JS, Wang BL, Fink GR, Stephanopoulos G. Xylose isomerase overexpression along with engineering of the pentose phosphate pathway and evolutionary engineering enable rapid xylose utilization and ethanol production by Saccharomyces cerevisiae. Metab Eng. 2012;14:611-22.

90. Lee SH, Kodaki T, Park YC, Seo JH. Effects of NADH-preferring xylose reductase expression on ethanol production from xylose in xylosemetabolizing recombinant Saccharomyces cerevisiae. J Biotechnol. 2012;158:184-91.

91. Valdehuesa KNG, Liu H, Ramos KRM, Park SJ, Nisola GM, Lee WK, Chung WJ. Direct bioconversion of $d$-xylose to 1,2,4-butanetriol in an engineered Escherichia coli. Process Biochem. 2014;49:25-32.

92. Koivistoinen OM, Kuivanen J, Barth D, Turkia H, Pitkanen JP, Penttila M, Richard P. Glycolic acid production in the engineered yeasts Saccharomyces cerevisiae and Kluyveromyces lactis. Microb Cell Fact. 2013;12:82-98.

93. Cabulong RB, Lee WK, Banares AB, Ramos KRM, Nisola GM, Valdehuesa KNG, Chung WJ. Engineering Escherichia coli for glycolic acid production from D-xylose through the Dahms pathway and glyoxylate bypass. Appl Microbiol Biotechnol. 2018;102:2179-89.

94. Cam Y, Alkim C, Trichez D, Trebosc V, Vax A, Bartolo F, Besse P, Francois $J M$, Walther T. Engineering of a synthetic metabolic pathway for the assimilation of (D)-xylose into value-added chemicals. ACS Synth Biol. 2016;5:607-18

\section{Publisher's Note}

Springer Nature remains neutral with regard to jurisdictional claims in published maps and institutional affiliations. 